\title{
USO DO AÇAFRÃO (Curcuma longa L.) NA REDUÇÃO DA Escherichia coli (ATCC 25922) E Enterobacter aerogenes (ATCC 13048) EM RICOTA ${ }^{1}$
}

\author{
The use of turmeric in the reduction of Escherichia coli (ATCC 25922) \\ and Enterobacter aerogenes (ATCC 13048) in ricotta
}

\author{
Sandra Ribeiro Maia ${ }^{1}$, Ana Cristina Ferreira ${ }^{2}$, Luiz Ronaldo de Abreu ${ }^{3}$
}

\begin{abstract}
RESUMO
Considerando o envolvimento de queijos como veículo de microrganismos patogênicos, foi avaliada a eficiência do extrato alcoólico de cúrcuma adicionado à ricota, na redução de Escherichia coli e Enterobacter aerogenes. Foram fabricados três lotes de ricota cremosa e inoculados com $10^{4} \mathrm{UFC} / \mathrm{mL}$ de Escherichia coli (ATCC 25922) e $10^{5} \mathrm{UFC} / \mathrm{mL}$ de Enterobacter aerogenes (ATCC 13048). Às ricotas, foram adicionados $0,4 \%$ de $\mathrm{NaCl}$ e extrato alcoólico de Curcuma longa $\mathrm{L}$., em concentrações que variaram de $0,0 \%$ a 2,0\%. As ricotas foram avaliadas físico-química e microbiologicamente em $0,1,7,14 \mathrm{e}$ 21 dias de armazenamento refrigerado. O percentual de umidade das ricotas foi, em média, de $73 \%$. O pH médio observado foi de 5,4 e o percentual de gordura de 3\%. Pelos resultados, evidenciou-se, após 21 dias, uma redução do número de Escherichia coli de aproximadamente dois ciclos logaritmicos nos tratamentos utilizados de $0,5 \%, 1,0 \%, 1,5 \%$ e $2,0 \%$ de cúrcuma. Já para Enterobacter aerogenes, a redução foi menor, de aproximadamente um ciclo logaritmico, de $10^{5} \mathrm{UFC} / \mathrm{mL}$ para $10^{4} \mathrm{UFC} / \mathrm{mL}$, também nos tratamentos utilizados de $0,5 \%, 1,0 \%, 1,5 \%$ e 2,0\% de cúrcuma. Apesar de os resultados evidenciarem uma redução do número de células viáveis dos microrganismos avaliados, a cúrcuma não deverá ser o único meio preservativo, considerando uma contaminação inicial de $10^{4} \mathrm{UFC} / \mathrm{mL}$ de Escherichia coli e $10^{5} \mathrm{UFC} / \mathrm{mL}$ de Enterobacter aerogenes, pois não atenderia à legislação vigente quanto aos requisitos microbiológicos para queijos.
\end{abstract}

Termos para indexação: Curcuma longa, Escherichia coli, Enterobacter aerogenes, ricota.

\begin{abstract}
Considering the cheese involvement as a vehicle of pathogenic microorganisms it was evaluated the eficciency of the ethanolic turmeric extract added to ricotta, in the reduction of Escherichia coli and Enterobacter aerogenes. Three lots of creamy ricotta were manufacturated and inoculated with $10^{4} \mathrm{UFC} / \mathrm{mL}$ of Escherichia coli (ATCC 25922) and $10^{5} \mathrm{UFC} / \mathrm{mL}$ of Enterobacter aerogenes (ATCC 13048). It was added $0,4 \%$ of $\mathrm{NaCl}$ and ethanolic turmeric extract into concentrations that varied from $0.0 \%$ to $2.0 \%$ into the ricotta. The ricottas were evaluated in $0,1,7,14$ and 21 days under refrigerated storage. The ricotta moist percentage was of $73 \%$ in average. The average $\mathrm{pH}$ observed was of 5,4 and the fat percentage was of $3 \%$. Microbiological results have shown a reduction in the number of Escherichia coli, after 21 days, about two logarithms cycles in treatments which $0.5 \%, 1.0 \%, 1.5 \%$ and $2.0 \%$ of ethanolic turmeric extract was used. As to Enterobacter aerogenes there was less reduction, at about one logarithm cycle, from $10^{5} \mathrm{UFC} / \mathrm{mL}$ to $10^{4} \mathrm{UFC} / \mathrm{mL}$, also in treatments in which $0.5 \%, 1.0 \%$, $1.5 \%$ and $2.0 \%$ of ethanolic turmeric extract was used. Although the results have shown a reduction in the number of the viable cells of the microorganisms evaluated, the ethanolic turmeric extract cannot be the single preservative means, considering a initial contamination of $10^{4} \mathrm{UFC} / \mathrm{mL}$ of Escherichia coli and $10^{5} \mathrm{UFC} / \mathrm{mL}$ of Enterobacter aerogenes for that would not be according to the current legislation referring to the microbiological cheese requirements.
\end{abstract}

INDEX TERMS: Turmeric, Escherichia coli, Enterobacter aerogenes, ricotta.

(Recebido para publicação em 28 de abril de 2003 e aprovado em 6 de agosto de 2003)

\section{INTRODUÇÃO}

Os condimentos são utilizados com a finalidade de realçar ou repor características, como a cor e o sabor, que com o processamento, podem ser perdidas. Existem cerca de 70 condimentos diferentes, cultivados e utilizados em todo o mundo.

\footnotetext{
1. Parte integrante da dissertação apresentada à Universidade Federal de Lavras/UFLA - Caixa Postal 37, 37200-000 - Lavras, MG, pelo primeiro autor, como requisito para obtenção do título de Mestre, área de concentração em Ciência dos Alimentos. smaia@cefetonline.com.br.

2. Tecnóloga em Laticínios, M.Sc. em Ciência dos Alimentos, DCA/UFLA. Professora da Escola Agrotécnica Federal de Inconfidentes - Inconfidentes, MG. 3. Professor, Dr. do Departamento de Ciência dos Alimentos/UFLA.
} 
Vários estudos conclusivos sobre os condimentos têm demonstrado que eles apresentam propriedades antimicrobianas, antioxidantes e medicinais, e existem evidências de que o aumento do consumo dos condimentos pode levar a uma mudança na microbiota intestinal, reduzindo a incidência de câncer. Sabe-se do efeito inibidor de determinados condimentos no crescimento de microrganismos deteriorantes e patogênicos veiculados por alimentos.

O uso de condimentos como conservante de alimentos é de grande interesse para os consumidores, pois não apresentam risco à saúde, mesmo quando empregados em quantidades relativamente altas. Admitese a perspectiva do uso de substâncias naturais presentes nos condimentos em substituição aos aditivos sintéticos utilizados no processamento dos alimentos com a finalidade de conservação. Conforme apontam alguns estudos, esses aditivos podem ser associados a doenças nos seres humanos. Cresce também o interesse por corantes naturais para a utilização na indústria de alimentos em substituição aos artificiais, em razão, principalmente, de estudos que apontam os efeitos tóxicos causados por corantes sintéticos (SAFFORD e GOODWIN, 1985).

Pela sua cor amarela, a cúrcuma tem atraído a atenção há muito tempo. O gênero Curcuma, pertencente à família Zingiberaceae, é constituído por cerca de 70 espécies rizomatosas de plantas herbáceas distribuídas por vários países, inclusive, América do Sul (MATHAI, 1979). A espécie Curcuma longa L. é a que possui maior importância comercial e utilização em alimentos (GOVINDARAJAN, 1980).

A cúrcuma, também conhecida por açafrão da terra, de cultivo relativamente simples, vem despertando um interesse cada vez maior, pela possibilidade de sua utilização em substituição a corantes sintéticos, especificamente a tartrazina. Somado a isso, a cúrcuma apresenta também atividade antimicrobiana, fato de grande interesse na indústria de alimentos, em substituição aos conservantes sintéticos.

Os rizomas maduros dessa planta contêm amido, óleo essencial e pigmentos corantes, entre esses, a curcumina, de cor amarelo-alaranjada, empregada em alimentos (PARRY, 1969).

Propriedades antioxidantes da cúrcuma foram investigadas (SRINIVAS et al., 1992; OSAWA, 1995; SEMWAL et al., 1997). O extrato alcoólico de cúrcuma inibiu o crescimento da maioria dos organismos em colicistites (LUTOMSKI et al., 1974). Os óleos essenciais apresentaram atividades bactericida (LUTOMSKI et al., 1974) e fungistática (SAWADA, 1971). Segundo Dhar (1968), o extrato alcoólico também exibiu atividade antiprotozoária. Em outras publicações, a cúrcuma, em suas várias apresentações, aparece associada à atividade antiparasítica (ARAÚJO, 1998, 1999), antiHIV (MAZUMBER, 1995) e antitumoral (HUANG, 1998).

A cúrcuma em pó é um constituinte indispensável do curry indiano, também utilizado em pasta de mostarda e em condimentos. É também usada em combinação com a páprica em muitos queijos processados e em produtos à base de gordura.

O interesse por queijos condimentados tem crescido. A ricota condimentada tem aparecido como uma boa opção de consumo, por se tratar de um alimento de fácil digestão e uma das formas mais simples e econômicas de aproveitamento do soro proveniente de vários tipos de queijos, obtendo-se um produto de fácil comercialização e baixo custo.

A ricota fresca é considerada um dos produtos que apresentam melhores condições para o desenvolvimento e crescimento de microrganismos, como Salmonella, Staphylococcus aureus, coliformes totais e fecais. Isso se deve, principalmente, à disponibilidade de nutrientes, como sais minerais e lactose, entre outros, o que compromete a qualidade do produto em sua vida de prateleira.

Em geral, a presença de coliformes em alimentos pode ser o indício de contaminação fecal, existindo, portanto, a possibilidade da presença de bactérias enteropatogênicas. Seu crescimento inviabilizaria o consumo desses alimentos (FRAZIER, 1967).

Nesse contexto, com este trabalho teve-se por objetivo verificar a eficiência da cúrcuma na redução da população de Escherichia coli e Enterobacter aerogenes na ricota.

\section{MATERIAL E MÉTODOS}

O experimento foi realizado no Laboratório de Microbiologia da Usina Piloto de Laticínios da Escola Agrotécnica Federal de Inconfidentes (EAFI), MG e no Laboratório de Laticínios do Departamento de Ciência dos Alimentos da Universidade Federal de Lavras (UFLA), MG.

$\mathrm{O}$ pó de cúrcuma, com teor de curcumina na ordem de $4,16 \%$, foi doado pela Escola de Agrononomia e Engenharia de Alimentos da Universidade Federal de Goiás. A determinação do teor de curcumina da cúrcu- 
ma foi realizada segundo método de ensaio da NBR 13624 da Associação Brasileira de Normas Técnicas (ABNT, 1996).

O solvente utilizado no preparo do extrato alcoólico foi o etanol, de uso comercial, a $95,3^{\circ} \mathrm{GL}$. O extrato alcoólico de cúrcuma foi preparado utilizando-se 100 g de pó de cúrcuma para $600 \mathrm{~mL}$ de etanol. O pó foi misturado ao etanol e deixado em repouso por 24 horas, quando foi, então, filtrado em papel de filtro. O pó foi descartado e utilizou-se o filtrado. O extrato alcoólico de cúrcuma foi submetido a teste de esterilidade e demonstrou ausência de microrganismos capazes de formar colônias em ágar, durante 48 horas de incubação a temperaturas de $37^{\circ}$ a $50^{\circ} \mathrm{C}$.

Os microrganismos usados para inoculação na ricota, padrão ATCC, na forma reativada, foram adquiridos na Fundação Tropical André Tozello, em Campinas, São Paulo. As linhagens adquiridas foram a Escherichia coli (ATCC 25922) e Enterobacter aerogenes (ATCC 13048).

Os meios de cultura utilizados foram o Chromocult Coliform Ágar e Caldo Nutritivo, marca MERCK.

O soro para fabricação da ricota foi proveniente da fabricação de queijo-de-minas frescal, produzido na usina piloto da EAFI, Inconfidentes, MG.

O soro fresco proveniente da fabricação de queijo-de-minas frescal foi quantificado e colocado em um tanque, que permitia o aquecimento com vapor direto, onde, então, foi reduzida a acidez original (situada em torno de $14^{\circ} \mathrm{D}$ ) para $8^{\circ} \mathrm{D}$, utilizando-se $\mathrm{NaOH}$.

$\mathrm{O}$ aquecimento com vapor direto foi feito até atingir a temperatura de $92^{\circ} \mathrm{C}$, sendo aí iniciada a acidificação, utilizando-se uma solução contendo $100 \mathrm{~mL}$ de ácido lático a 85\% para cada 100 litros de soro, diluído em volume de água 10 vezes superior.

$\mathrm{O}$ aquecimento foi interrompido próximo de $95^{\circ} \mathrm{C}$, aguardando-se o tempo necessário até que a massa estivesse formada na superfície do soro, procedendose a seguir à coleta com dessoradores.

A massa foi depositada em um recipiente esterilizado e levada para resfriar em câmara de fluxo laminar, sendo, em seguida, armazenada a $5^{\circ} \mathrm{C}$ para posterior utilização.

$\mathrm{O}$ experimento foi realizado em esquema fatorial $5 \times 5 \times 3$, sendo cinco concentrações do extrato alcoólico de cúrcuma, incluindo o controle, cinco períodos de estocagem e três repetições. As embalagens das amostras foram esterilizadas e, em cada uma, adicionaram- se $25 \mathrm{~g}$ da ricota resfriada a $5^{\circ} \mathrm{C}, 0,4 \%(0,1 \mathrm{~g})$ de $\mathrm{NaCl}$ e o extrato alcoólico de cúrcuma, nas concentrações de $0,0 \%, 0,5 \%$ (0,74 mL), 1,0\% (1,47 mL), 1,5\% (2,21 $\mathrm{mL})$ e $2,0 \%(2,94 \mathrm{~mL})$, procedendo-se à agitação para que os componentes se misturassem.

O extrato alcoólico de cúrcuma foi preparado na proporção de 1:6 (cúrcuma e álcool a 95,3ํㅡ). Após a inoculação, os potes foram fechados e encaminhados à câmara fria e mantidos a $5^{\circ} \mathrm{C}$ durante 21 dias.

Amostras foram coletadas para análises nos dias 0, 1, 7, 14 e 21, conforme a Figura 1.

Para o preparo do inóculo, foi retirada do tubo slant uma alçada de cada cultura ativa de Enterobacter aerogenes e Escherichia coli e colocada em um tubo de ensaio de $5 \mathrm{~mL}$ contendo caldo infusão de cérebro e coração.

As culturas foram incubadas e, a partir de 30 minutos até 2 horas, foi realizado o plaqueamento em 4 diluições a cada intervalo de 30 minutos para determinação da concentração das células. A determinação da concentração das células de Enterobacter aerogenes e Escherichia coli em BHI foi realizada de forma simultânea por contagem em placas (pour-plate), utilizandose o meio de cultura Chromocult Coliform Ágar (Merck) com incubação a $37^{\circ} \mathrm{C}$, por 24 horas.

A inoculação foi feita imediatamente após a mistura da ricota com o extrato alcoólico de cúrcuma, nas concentrações de $0,5 \%, 1,0 \%, 1,5 \%$ e $2,0 \%$, sempre com $0,4 \%$ de $\mathrm{NaCl}$. Também foi inoculada uma das amostras sem o extrato alcoólico de cúrcuma, que foi denominado controle. A inoculação de Escherichia coli e Enterobacter aerogenes na ricota foi na ordem de $10^{4}$ $\mathrm{UFC} / \mathrm{mL}$ e $10^{5} \mathrm{UFC} / \mathrm{mL}$, respectivamente. Essa concentração foi obtida a partir da incubação das duas culturas por 30 minutos a $37^{\circ} \mathrm{C}$.

Foram realizadas análises físico-químicas do soro: $\mathrm{pH}$, acidez titulável e gordura, e da ricota: $\mathrm{pH}$, teor de umidade e gordura. A ricota foi analisada no dia da fabricação, em todas as repetições.

Todas as análises microbiológicas foram realizadas em duplicatas. A contagem em placas (pour-plate), com identificação simultânea de Enterobacter aerogenes e Escherichia coli, foram feitas utilizando-se o meio de cultura Chromocult Coliform Ágar com incubação a $37^{\circ} \mathrm{C}$, por 24 horas (MERCK). Nesse método, as colônias são diferenciadas pela cor. Para Enterobacter aerogenes, a coloração observada é vermelho-salmão e para Escherichia coli, colônias azuis- escuras ou violeta. 


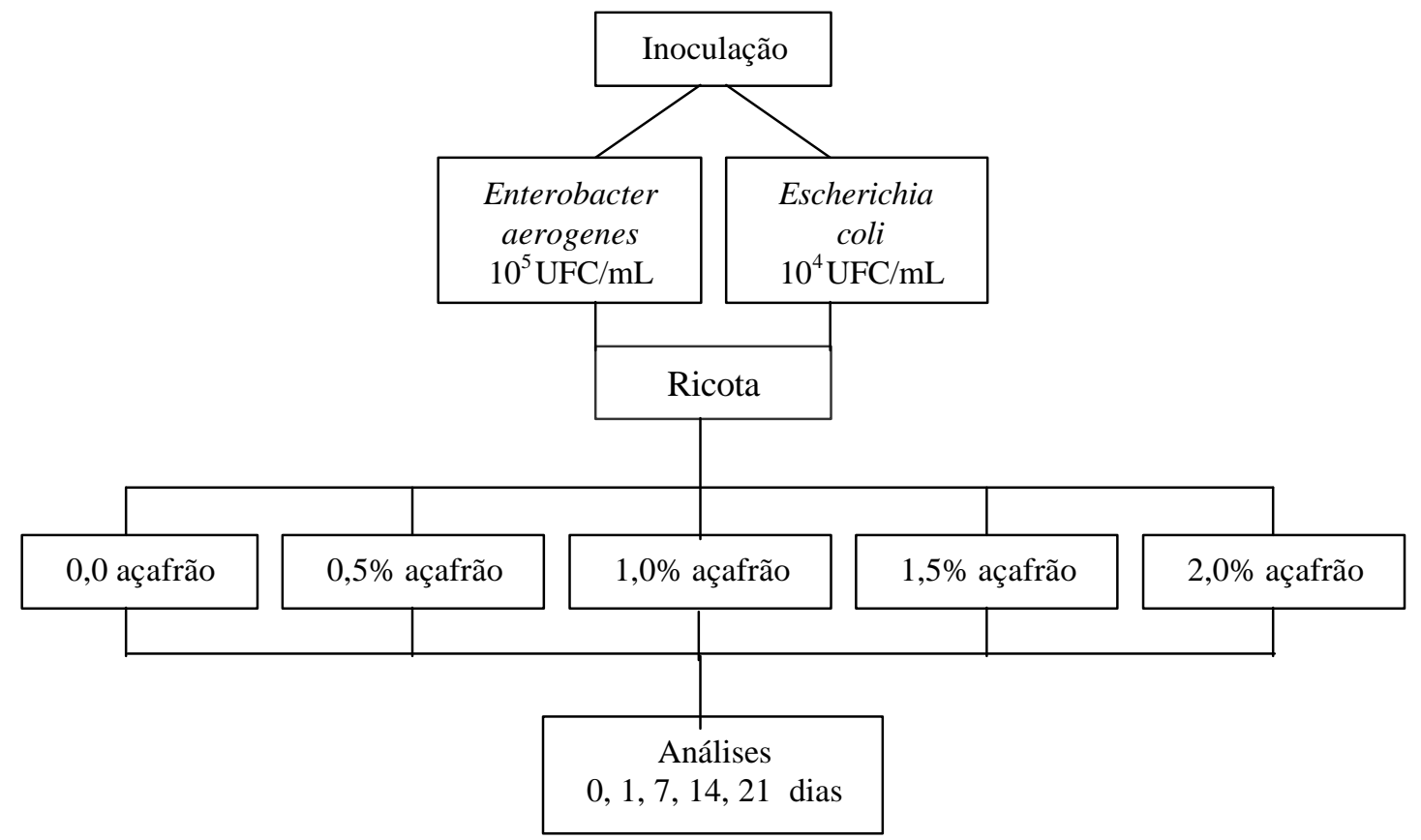

FIGURA 1 - Tratamentos aplicados e análises realizadas.

\section{RESULTADOS E DISCUSSÃO}

A determinação das populações apresentada nas Tabelas 1 e 2 demonstra que foram inoculados, em média, nos três lotes de ricota, $8,2.10^{4} \mathrm{UFC} / \mathrm{mL}$ de $E s$ cherichia coli (ATCC 25922) e 9,6.105 UFC/mL de Enterobacter aerogenes (ATCC 13048), respectivamente, no dia da fabricação.

Após 21 dias de tratamento, ocorreu uma redução do número de células de Enterobacter aerogenes de um ciclo logaritmico, se comparado ao controle do dia da fabricação, quando o número de células viáveis era de aproximadamente 9,6.10 U.F.C./mL. Nesse período, o número de células viáveis foi reduzido a valores aproximados de $10^{4}$, nos tratamentos aplicados de $0,5 \%, 1,0 \%, 1,5 \%$, e $2,0 \%$ do extrato alcoólico de cúrcuma.

Conforme resultados apresentados na Figura 2, em nenhum dos dias avaliados o número Enterobacter aerogenes na ricota tratada com extrato alcoólico de cúrcuma, em nenhuma concentração, foi superior ao controle do dia da fabricação ou ao controle de cada dia avaliado.

Foram observadas reduções nas contagens de Enterobacter aerogenes com relação aos teores do extrato alcoólico de cúrcuma aplicados, mas os valores de $1,0 \%, 1,5 \%$ e $2,0 \%$ apresentaram melhores resultados durante os primeiros 14 dias de armazenamento. De acordo com Shelef (1983), as concentrações de condimento utilizadas com a finalidade de inibir o crescimento bacteriano variam de $1,0 \%$ a $5,0 \%$.

A redução brusca do número de células do controle de 7 a 14 dias de armazenamento poderia ser atribuída a um decréscimo normal do número de células, característico de uma curva normal de crescimento bacteriano, quando os metabólitos decorrentes do próprio metabolismo dos coliformes concorreriam para essa redução. Os coliformes fermentam a lactose com produção de ácidos. 
TABELA 1 - Contagem média de Escherichia coli na ricota tratada com extrato alcoólico de cúrcuma nas concentrações de 0,0 a $2,0 \%$.

\begin{tabular}{cccccc}
\hline \multirow{2}{*}{$\begin{array}{c}\text { Tratamento } \\
\text { (\% Cúrcuma) }\end{array}$} & $\mathbf{5}$ & \multicolumn{5}{c}{ Armazenamento (dias) } \\
\cline { 2 - 6 } & $\mathbf{0}$ & $\mathbf{1}$ & $\mathbf{7}$ & $\mathbf{1 4}$ & $\mathbf{2 1}$ \\
\hline $0,0 \%$ & $8,2.10^{4}$ & $5,8.10^{5}$ & $1,4.10^{4}$ & $9,9.10^{3}$ & $2,4.10^{3}$ \\
$0,5 \%$ & $8,2.10^{4}$ & $6,4.10^{4}$ & $1,6.10^{4}$ & $3,6.10^{3}$ & $4,6.10^{2}$ \\
$1,0 \%$ & $8,2.10^{4}$ & $3,8.10^{4}$ & $1,6.10^{4}$ & $4,5.10^{3}$ & $8,8.10^{2}$ \\
$1,5 \%$ & $8,2.10^{4}$ & $1,6.10^{4}$ & $3,8.10^{4}$ & $2,0.10^{2}$ & $4,7.10^{2}$ \\
$2,0 \%$ & $8,2.10^{4}$ & $1,1.10^{4}$ & $4,4.10^{4}$ & $6,3.10^{2}$ & $1,8.10^{2}$ \\
\hline
\end{tabular}

U.F.C. - Contagem de unidades formadoras de colônias

TABELA 2 - Contagem média de Enterobacter aerogenes na ricota tratada com extrato alcoólico de cúrcuma nas concentrações de 0,0 a $2,0 \%$.

\begin{tabular}{cccccc}
\hline \multirow{2}{*}{$\begin{array}{c}\text { Tratamento } \\
\text { (\% Cúrcuma) }\end{array}$} & $\mathbf{5}$ & \multicolumn{5}{c}{ Armazenamento (dias) } \\
\cline { 2 - 6 } & $\mathbf{0}$ & $\mathbf{1}$ & $\mathbf{7}$ & $\mathbf{1 4}$ & $\mathbf{2 1}$ \\
\hline $0,0 \%$ & $9,6.10^{5}$ & $1,2.10^{6}$ & $5,3.10^{6}$ & $1,2.10^{5}$ & $3,2.10^{5}$ \\
$0,5 \%$ & $9,6.10^{5}$ & $6,0.10^{5}$ & $4,6.10^{5}$ & $8,2.10^{4}$ & $8,0.10^{3^{*}}$ \\
$1,0 \%$ & $9,6.10^{5}$ & $4,6.10^{5}$ & $1,0.10^{5}$ & $6,4.10^{4}$ & $2,5.10^{4}$ \\
$1,5 \%$ & $9,6.10^{5}$ & $5,4.10^{5}$ & $1,5.10^{5}$ & $7,2.10^{4}$ & $3,9.10^{4}$ \\
$2,0 \%$ & $9,6.10^{5}$ & $3,9.10^{5}$ & $6,3.10^{4}$ & $5,0.10^{4}$ & $4,7.10^{4}$ \\
\hline
\end{tabular}

U.F.C. - Contagem de unidades formadoras de colônias

*População estimada

Pelas análises de Escherichia coli, demonstrouse que, após 21 dias de tratamento, ocorreu uma redução do número de Escherichia coli de aproximadamente dois ciclos logaritmicos, se comparado ao controle do dia da fabricação, quando o número de células viáveis foi de, em média, $8,2.10^{4}$ U.F.C./mL. Nesse período, o número de células viáveis foi reduzido a valores de $10^{2}$, nos tratamentos aplicados de $0,5 \%, 1,0 \%, 1,5 \%$, e 2,0\% do extrato alcoólico de cúrcuma, e no controle, o decréscimo foi de um ciclo logaritmico.

Conforme resultados apresentados na Figura 3, um ligeiro incremento do número de células viáveis de Escherichia coli, quando comparado ao controle, de 1 para 7 dias de armazenamento, principalmente considerando os tratamentos mais altos $(1,5 \%$ e $2,0 \%$ do extrato alcoólico de cúrcuma), poderia ser atribuído a uma maior disponibilidade de nutrientes no início do perío- do de incubação. Isso porque a cúrcuma também contém nutrientes que poderiam servir de substrato para a bactéria. Com os tratamentos aplicados de $0,5 \%$ e $1,0 \%$ de cúrcuma de 1 para 7 dias de armazenamento, o controle do número de Escherichia coli foi mantido, seguindo essa tendência de queda até 21 dias.

Visualmente, parece que as bactérias ainda não estavam na fase exponencial de multiplicação quando foram inoculadas, e sim na fase de adaptação. Nas amostras tratadas, foram mais rapidamente inibidas, quando comparadas ao controle.

Conforme ainda resultados apresentados na Figura 3, à exceção do dia 7 , em nenhum outro dia avaliado a contagem de Escherichia coli em ricota tratada com extrato alcoólico de cúrcuma superou o controle do dia da fabricação ou ao controle de cada dia avaliado. 


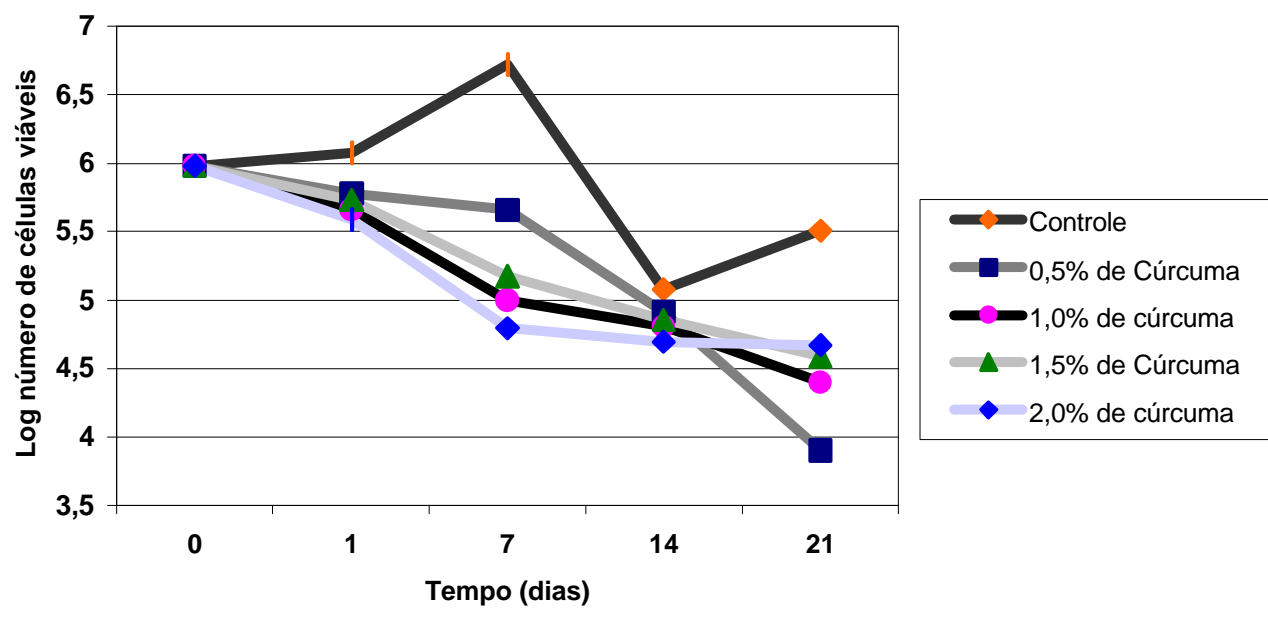

FIGURA 2 - Contagens médias (Log UFC/mL) de Enterobacter aerogenes em ricota tratada com extrato alcoólico de cúrcuma nas concentrações de $0,0 \%$ a $2,0 \%$.

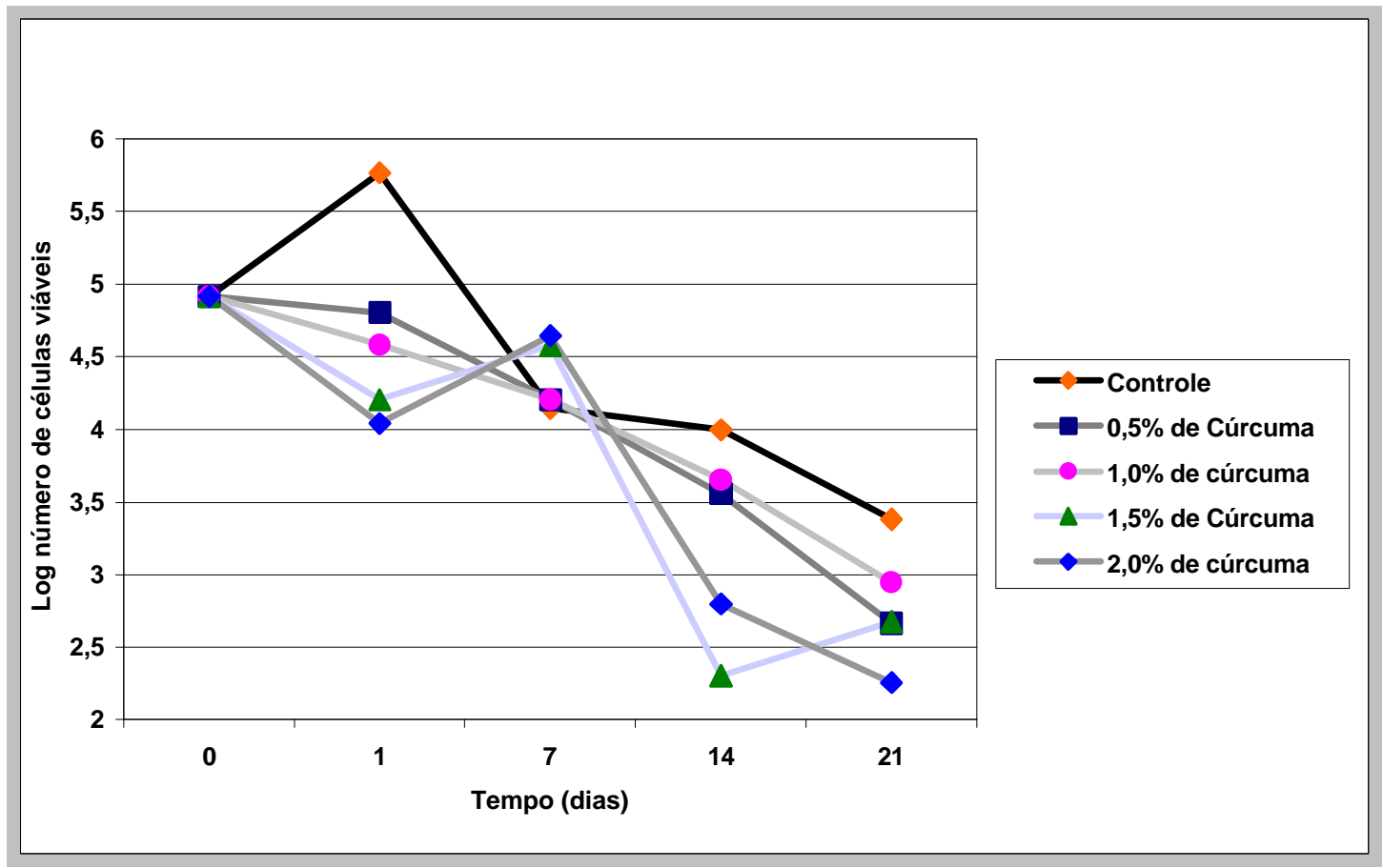

FIGURA 3 - Contagens médias (Log UFC/mL) de Escherichia coli em ricota tratada com extrato alcoólico de cúrcuma nas concentrações de $0,0 \%$ a $2,0 \%$.

Ciênc. agrotec., Lavras, v. 28, n. 2, p. 358-365, mar./abr., 2004 
Nenhuma das amostras de ricota, independente do tipo de tratamento e dia de análise, estavam dentro dos padrões preconizados pelo Ministério da Agricultura, para contagem de coliformes totais (Enterobacter aerogenes) e para Escherichia coli.

De acordo com o regulamento, os critérios de aceitação para coliformes totais são duas amostras com valores entre 100 e 1000 coliformes/g e os valores mínimos encontrados foram da ordem de $10^{4}$. Para Escherichia coli, em duas amostras, os valores devem estar entre 50 e 500 coliformes/g e os valores mínimos encontrados foram da ordem de $10^{2}$.

\section{CONCLUSÃO}

O extrato alcoólico de cúrcuma nas concentrações de $0,5 \%, 1,0 \%, 1,5 \%$ e $2,0 \%$ possui a propriedade de reduzir o número de Escherichia coli e Enterobacter aerogenes inoculadas na ricota.

Apesar de pelos resultados se evidenciar uma redução do número de células viáveis dos microrganismos avaliados, a cúrcuma não deverá ser o único meio preservativo, considerando uma contaminação inicial de $10^{4} \mathrm{UFC} / \mathrm{mL}$ de Escherichia coli e $10^{5} \mathrm{UFC} / \mathrm{mL}$ de Enterobacter aerogenes, pois não atenderia à legislação vigente quanto aos requisitos microbiológicos para queijos.

A combinação do uso da cúrcuma como aditivo natural na redução de Escherichia coli e Enterobacter aerogenes em ricota, associada a outras técnicas com a finalidade de conservação de alimentos, é recomendada.

\section{REFERÊNCIAS BIBLIOGRÁFICAS}

ASSOCIAÇÃO BRASILEIRA DE NORMAS TÉCNICAS. NBR 13624: cúrcuma: determinação do teor de curcumina: método de ensaio. Rio de Janeiro, 1996.

ARAÚJO, C. A. C. Leishmania amazonensis: in vivo experiments with diarylheptanoids from Leguminosae and Zingiberaceae plants. Memórias Instituto Oswaldo Cruz, Rio de Janeiro, v. 93, p. 306, 1998. Suplemento 2 .

ARAÚJO, C. A. C. Studies on the effectiveness of diarylheptanoids derivates against Leishmania amazonensis. Memórias Instituto Oswaldo Cruz, Rio de Janeiro, v. 94, p. 791-794, 1999.
DHAR, M. L. C. Screening of indian plants for biological activity I. Indian Journal of Experimental Biology, New Delhi, v. 6, p. 232-240, 1968.

FRAZIER, W. C. Food microbiology. 2. ed. New York: Mc Graw-Hill, 1967. 537 p.

GOVINDARAJAN, V. S. Turmeric: chemistry, technology and quality. CRC Critical Reviews in Food Science and Nutrition, Cleveland, v. 12, n. 3, p. 199-301, 1980.

HUANG, M. T. Inhibitory effect of curcumin, chlorogenic acid, caffeic acid and ferulic acid on tumor promotion in mouse skin by 12-O-tetradecanoyl phorbol-13-acetate. Cancer Research, Baltimore, [S.1.], v. 48, p. 5941-5946, 1998.

LUTOMSKI, J.; KEDZIA, B.; DEBSKA, W. Effect of na alcohol extract and active ingredients from Curcuma longa on bacteria and fungi. Planta Medica, Stuttgart, v. 26, n. 1 , p. 9, 1974.

MATHAI, C. K. The pattern of rhizome yield and their accumulation of commercially important chemical constituints in turmeric (Curcuma spices) during growth and development. Qualitas Plantarum Plant Foods For Human Nutrition, The Hague, v. 28, n. 3, p. 219-225, 1979.

MAZUMBER, A. Inhibition of human immunodeficiency virus type-1 integrase by curcumin. Biochemical Pharmacology, New York, v. 49, p. 1165-1170, 1995.

OSAWA, T. Antioxidative activity of tetrahydrocurcuminoids. Bioscience Biotech. Biochemical, [S.1.], v. 59, p. 1609-1612, 1995.

PARRY, J. W. Spices: morphology, histology, chemistry. New York: Chemical, 1969. v. 2, p. 74-78.

SAFFORD, R. J.; GOODWIN, B. F. J. Immunological studies on tartrazine. International Archives of Allergy and Applied Immunology, Basel, v. 77, n. 3, p. 331-336, 1985.

SAWADA, T. Evaluation of crude drugs by bioassay, III: comparison with local variation of the contents and the fungistatic action of the essential oil from the roots of Curcuma longa. Shoyakugaku Zasshi, Kyoto, v. 25, n. 1 , p. 11, 1971. 
SEMWAL, A. D.; SHARMA, G. K.; ARYA, S. S. Antioxygenic activity of turmeric (Curcuma longa) in sunflower oil and Ghec. Journal of Food Science and Technology, Mysore, v. 34, n. 1, p. 67-69, 1997.

SHELEF, L. A. Antimicrobial affects of spices. Journal of Food Safety, Connecticut, v. 6, n. 1, p. 29-44, Aug. 1983.
SRINIVAS, L.; SHALINI, V. K.; SHYLAJA, M. Turmerin: a water soluble antioxidant peptide from turmeric (Curcuma longa). Archive Biochemistry Biophisic, Mysore, v. 292, n. 2, p. 617-627, 1992. 